\title{
Genomic analysis of Vavilov's historic chickpea landraces using GWAS, AMMI and GGE biplot analyses
}

\author{
Alena Sokolkova \\ Peter the Great St. Petersburg \\ Polytechnic University, St. Petersburg, \\ Russia \\ alyonasok@yandex.ru \\ Noelia Carrasquila-Garcia \\ University of California Davis, \\ Department of Plant Pathology, Davis, \\ CA 95616 USA \\ noecarras@ucdavis.edu \\ Douglas R. Cook \\ University of California Davis, \\ Department of Plant Pathology, Davis, \\ CA 95616 USA \\ drcook@ucdavis.edu
}

\author{
Sergey V. Bulyntsev \\ Federal Research Centre All-Russian \\ N.I. Vavilov Institute of Plant Genetic \\ Resources (VIR), St. Petersburg, Russia \\ s_bulyntsev@mail.ru \\ Eric von Wettberg \\ University of Vermont, Department of \\ Plant and Soil Science, Burlington, VT \\ 05405, USA \\ College of Letters \\ eric.bishop-von-wettberg@uvm.edu \\ Sergey V. Nuzhdin \\ University of Southern California, \\ Program in Molecular and \\ Computational Biology, Dornsife Arts \\ \& Sciences, Los Angeles, CA 90089 \\ USA \\ snuzhdin@usc.edu
}

\author{
Peter L. Chang \\ University of Southern California, \\ Program in Molecular and \\ Computational Biology, Dornsife \\ College of Letters Arts \& Sciences, Los \\ Angeles, CA 90089 USA \\ peterc@usc.edu \\ Margarita A. Vishnyakova \\ Federal Research Centre All-Russian \\ N.I. Vavilov Institute of Plant Genetic \\ Resources (VIR), St. Petersburg, Russia \\ m.vishnyakova@vir.nw.ru \\ Maria G. Samsonova \\ Peter the Great St. Petersburg \\ Polytechnic University, Department of \\ Applied Mathematics, St. Petersburg, \\ Russia \\ m.g.samsonova@gmail.com
}

\begin{abstract}
The Vavilov seed bank contains numerous landraces collected nearly hundred years ago, before intensive breeding of most crops, and thus is a potential reservoir of historic crop adaptations. Here we analyze the genomes of 407 of Vavilov's original landraces, sampled from major historic centers of chickpea cultivation and secondary diversification. We performed GWAS to find associations between VIR's chickpea accessions and phenotypic data obtained in Kuban experimental station of VIR in 2017 under infectious conditions. To find out the effects of infectious conditions on phenotypic traits, the data was subjected to AMMI and GGE biplot analyses.
\end{abstract}

Keywords - chickpea (Cicer arietinum L.), GWAS analysis, candidate genes, AMMI, GGE biplot

\section{Motivation and Aim}

The Vavilov seed bank contains numerous landraces collected nearly hundred years ago, before intensive breeding of most crops, and thus is a potential reservoir of historic crop adaptations. The expanded diversity of these early collections likely contains 'genetic gems' with the potential to enhance modern breeding efforts [1]. Here we analyze the genomes of 407 of Vavilov's original landraces, sampled from major historic centers of chickpea cultivation and secondary diversification.

Earlier we have performed Genome-wide association study (GWAS) to find associations between VIR's chickpea accessions and phenotypic data obtained in Kuban experimental station of VIR in 2016. GWAS analysis identified a large number of genome intervals and potential gene candidates that may affect important agronomic traits. In 2017 the phenotypic data was obtained in Kuban experimental station of VIR under infectious conditions. GWAS analysis was performed with phenotypic data obtained in 2017 and AMMI (Additive Main effects and Multiplicative Interaction) and GGE (Genotype plus Genotype-Environment interaction) biplot analyses were performed to determine the effect of genotype, environment and their interaction for phenotypic traits measured both in 2016 and 2017 under different infectious conditions.

\section{Methods}

Genotyping by sequencing (GBS) of VIR's 407 chickpea accessions from different countries identified 56,855 segregating single nucleotide polymorphisms (SNP). For these SNP calls we implemented inclusion criteria: minor allele frequency (MAF) more than 3\%, genotype call-rate more than 90\% . 2,579 SNPs passed all filters and remained for further analysis. We performed GWAS to find associations between VIR's chickpea accessions and phenotypic data obtained in Kuban experimental station of VIR in 2017 under infectious conditions. To find out the effects of infectious conditions on phenotypic traits, the data was subjected to AMMI and GGE biplot analyses.

\section{Results}

GWAS analysis identified three SNPs on chromosomes 2, 7 and 8, respectively, significally associated with days from begginning to end of flowering under infectious conditions. SNP on chromosome 8 was also detected in previous GWAS analysis performed with phenotypic traits obtained in 2016 and with specific climatic variables at collection sites [2]. This SNP is a strong candidate marker for future selection. The results indicated that AMMI and GGE biplot analyses are informative methods to evaluate the effects of infectious conditions on phenotypic traits.

\section{ACKNOWLEDGMENT}

Supported by the RSCF (16-16-00007).

\section{REFERENCES}

[1] Plekhanova E. et al. (2017) Genomic and phenotypic analysis of Vavilov's historic landraces reveals the impact of environment and genomic islands of agronomic traits. Sci. Rep. 7:4816. doi: 10.1038/s41598-017-05087-5.

[2] Sokolkova A.B. et al. (2020) Signatures of ecological adaptation in genomes of chickpea landraces. Biofizika. 65(2): 1-4. 